\title{
An Approach for Real-Time Database Modeling and Performance Management *
}

\author{
Jisu Oh and Kyoung-Don Kang \\ Department of Computer Science \\ State University of New York at Binghamton \\ $\{$ joh,kang\}@cs.binghamton.edu
}

\begin{abstract}
It is challenging to manage the performance of real-time databases (RTDBs) that are often used in data-intensive real-time applications such as agile manufacturing and target tracking. Feedback control has recently been considered a promising approach to enabling reliable real-time data service. However, most existing work on feedback control of RTDB performance is not based on a RTDB-specific control model, which is critical for closed-loop system design. To address this problem, we design a novel RTDB model that can capture RTDB dynamics by modeling the relation between the total arrival rate (sum of the transaction arrival rate and restart rate) and utilization via a difference equation. Based on the model, we design and tune a utilization controller to compute the total arrival rate adjustment needed to support the desired average/transient utilization. We also design a QoS management scheme and admission control technique that can judiciously adapt the transaction $Q o S$ and arrival rate in a RTDB, if necessary, to support the desired utilization, while enhancing the success ratio. In a simulation study, we show that our approach can support the desired average/transient utilization for a range of transaction arrival rates under severe data contention, while considerably improving the success ratio compared to the tested baselines.
\end{abstract}

\section{Introduction}

A number of real-time applications, including agile manufacturing, target tracking, and traffic control, are dataintensive. Real-time databases (RTDBs) are often used in these applications to process transactions within their deadlines using fresh sensor data reflecting the current real world status [5, 25, 26, 31]. Non-real-time databases are inappropriate for these applications as they are unaware of timing and data freshness constraints.

\footnotetext{
${ }^{*}$ This work was supported, in part, by a NSF grant CNS-0614771.
}

RTDB performance management is challenging due to dynamic data/resource contention. To address the problem, initial work including $[4,5,10]$ has applied feedback control theory to RTDB performance management. However, most existing work on feedback control of the RTDB performance (including $[4,5,10]$ ) is not based on a RTDBspecific system model, which is critical for the closed-loop system design. Instead, their system models are based on the fluid model $[9,24]$ originally adopted for scheduling real-time tasks involving no data conflicts $[16,18]$. Feedback control has been well studied for real-time scheduling and web QoS management in dynamic environments since the early work $[2,15,17,16]$. However, these approaches are not directly applicable to RTDBs as they do not consider data conflicts specific to databases, which can result in transaction aborts and restarts.

In this paper, we propose a novel approach called FACT (Feedback control of Arrivals and Conflicts for Timeliness). FACT directly considers data/resource contention for RTDB modeling. Unlike most existing work such as $[4,5,10]$, it requires no knowledge of (estimated) transaction execution times, which are hard to predict due to transaction aborts and restarts $[25,26]$. Specifically, we model RTDB dynamics by deriving the relation between the total arrival rate, i.e., transaction arrival rate + restart rate, and the utilization to represent the possible utilization increase (or decrease) due to varying degrees of data/resource contention ${ }^{1}$. A model-based design technique $[9,24]$ is used to mathematically model the relation between the total arrival rate and utilization. Based on the RTDB model, we design and tune a utilization controller to compute the total arrival rate adjustment necessary to support the desired target utilization at every sampling period. We also develop a transaction QoS adaptation scheme based on the milestone approach $[13,29]$ and adaptive admission control technique to support the target utilization according to the required total arrival rate adjustment computed in

\footnotetext{
${ }^{1}$ We use the terminology total arrival rate, since the restart of a transaction already in the system could affect the workload in a way similar to a new transaction arrival from the system's perspective.
} 
the feedback loop, while improving the success ratio under overload.

Without transactions supported by RTDBs, dataintensive real-time applications should manually maintain the temporal and logical consistency of sensor and nonsensor data, possibly producing wrong results based on stale/inconsistent sensor/non-sensor data [25, 26]. Unless transactions are executed serially, no concurrency control protocol can completely eliminate transaction aborts/restarts [7]. Thus, our approach is generally applicable to concurrent real-time transaction processing. To show the applicability of our approach, we consider both lockbased and optimistic concurrency control methods-two major approaches for concurrency control-for performance evaluation. Multi-version concurrency control could further reduce data conflicts in RTDBs. A thorough investigation is reserved for future work.

For performance analysis, we have performed a comprehensive simulation study for various arrival rates under severe data contention. In general, FACT has outperformed the tested baselines often used for RTDB performance comparisons $[4,5,10,11]$. With no knowledge of the transaction execution time, FACT can closely support the desired average and transient utilization, while considerably enhancing the success ratio compared to the baselines under overload. From this, we observe the applicability of our RTDB model and performance management techniques to reliable real-time data service.

The remainder of this paper is organized as follows. Several performance metrics for real-time data management have been described in Section 2. Our RTDB model and utilization controller are derived in Section 3. A RTDB architecture supporting transaction scheduling, concurrency control, feedback control, QoS management, and admission control is described in Section 4. Performance evaluation is given in Section 5. Related work is discussed in Section 6. Finally, Section 7 concludes the paper and discusses the future work.

\section{Deadline Semantics and Performance Met- rics}

In this paper, we consider firm deadline semantics; a real-time transaction is aborted and removed from the system upon its deadline miss to avoid wasting resources to execute tardy transactions. We assume that there are two types of transactions, i.e., sensor transactions and user transactions, similar to $[5,25,11,31]$. Each sensor transaction is a write-only transaction dedicated to periodically updating one specific sensor data object to maintain its temporal consistency. Thus, the number of sensor transactions is equal to the number of the sensor data objects in the system. User transactions are aperiodic transactions that can read/write non-sensor data but they can only read sensor data.

We measure the RTDB performance via the success ratio, CPU utilization, and $\operatorname{QoS}$ (Quality of Service) received by timely transactions that commit before their deadlines. The success ratio (SR) is defined as follows:

$$
S R=100 \times \frac{N_{\text {timely }}}{N_{\text {submitted }}}(\%)
$$

where $N_{\text {timely }}$ is the number of transactions committed before their deadlines and $N_{\text {submitted }}$ is the total number of transactions submitted to the RTDB. Thus, the SR indicates what fraction of the submitted transaction can finish within their deadlines. Note that the SR cannot be used as a setpoint for feedback control, since $N_{\text {submitted }}$ can continuously increase as the workload increases. Hence, it is impossible to support a fixed target success ratio for dynamic workloads. Instead, in this paper, we specify the target utilization for feedback control in a RTDB. As long as we fully utilize the CPU without saturating it, we can maximize the success ratio ${ }^{2}$. It is also known that the relationship between the load applied to the system and utilization can be modeled in a piecewise linear manner $[18,9]$ as long as the utilization is not saturated at $100 \%$. We further reduce the complexity of RTDB modeling by requiring no a priori knowledge of estimated transaction execution times.

In addition to the average utilization, we measure the transient utilization in terms of overshoot and settling time $[24,9]$. This is important because, for example, a RTDB can be overloaded due to substantial transient data conflicts and resulting transaction aborts and restarts in a short time interval. In this paper, we apply feedback control to control both the average and transient utilization. We set the sampling period for feedback control $S P=1 s$ as specified in Table 1 . We set a range of the target utilization $U_{t}[90 \%$, 95\%] to process as many transactions as possible within their deadlines, while avoiding overshoots under overload. Specifically, FACT initializes $U_{t}=90 \%$. It increments $U_{t}$ if $S R \geq 99 \%$ in a sampling period, which indicates the system is currently underutilized. It resets $U_{t}$ to $90 \%$ as soon as $S R<99 \%$. (A more sophisticated approach for $U_{t}$ adaptation is reserved for future work.) It is known that the system performance can be improved by adjusting the set-point according to the current workload [9].

To achieve a high success ratio, the target utilization $U_{t}$ can be dynamically increased up to $95 \%$ in our approach. Thus, it is critical to minimize an overshoot, if any, to avoid possible controller saturation at $100 \%$ utilization. For example, a 5\% overshoot saturates the controller when $U_{t}=95 \%$. A low $U_{t}$, e.g., $80 \%$, can be more resilient to high overshoots. However, it may considerably reduce the

\footnotetext{
${ }^{2}$ In this paper, we consider a main memory database model, in which the CPU is considered the main system resource, similar to $[28,6,31,5$, $10]$.
} 
Table 1. Desired Feedback Control Performance

\begin{tabular}{l|l|l}
\hline Notation & Description & Desired Value \\
\hline $\mathrm{SP}$ & Sampling Period & $1 \mathrm{~s}$ \\
$U_{t}$ & Target Utilization & {$[90 \%, 95 \%]$} \\
$M_{p}$ & Overshoot & $1 \%$ \\
$K_{s}$ & Settling Time & $250 \mathrm{~s}$ \\
\hline
\end{tabular}

success ratio. For these reasons, we require an overshoot $M_{p} \leq 1 \%$ in this paper. Since $M_{p} \leq 1 \%$, the relatively long settling time $K_{s}$, which has trade-off relations with $M_{p}$, does not significantly affect the RTDB performance.

Imprecise approaches have successfully been applied to real-time transaction processing. Examples include [29, 4, $5,21]$. Our QoS adaptation approach is similar to APPROXIMATE [29], which can monotonically increase the accuracy of the answer to a real-time query by executing it longer, while returning an approximate result, if necessary, to meet the deadline. Specifically, we assume that a transaction $T_{i}$ consists of one mandatory part $T_{i}^{m}$ and $p(\geq 1)$ optional parts $T_{i, 1}^{o}, \ldots, T_{i, p}^{o} . T_{i}^{m}$ has to be always executed to support the required minimum QoS level for transaction $T_{i}$, while $T_{i, j}^{o}(1 \leq j \leq p)$ only refines the results and can be skipped, if necessary, to improve the success ratio under overload. Therefore, the QoS level of transaction $T_{i}$ is:

$$
Q\left(T_{i}\right)=\frac{1+N_{i, \text { Eopt }}}{1+N_{i, \text { opt }}}
$$

where $N_{i, \text { Eopt }}$ and $N_{i, \text { opt }}$ represent the number of the executed optional parts and the total number of the optional parts in $T_{i}$. Thus, $0 \leq Q\left(T_{i}\right) \leq 1$. From this, we define the average QoS in a RTDB:

$$
Q o S=100 \times \sum_{T_{i} \in S_{\text {timely }}} \frac{Q\left(T_{i}\right)}{N_{\text {timely }}}(\%)
$$

where $S_{\text {timely }}$ is the set of transactions completed within their deadlines and $N_{\text {timely }}$ is the cardinality of $S_{\text {timely }}$.

\section{$3 \quad$ RTDB Modeling and Controller Design}

In this section, we propose a RTDB model that considers not only transaction arrivals but also restarts. Section 3.1 describes our open-loop RTDB model in terms of total arrival rate and utilization. In Section 3.2, the RTDB model parameters are derived via system identification. Based on the results, the closed-loop transfer function is derived in Section 3.3. Given the closed-loop transfer function, the controller is tuned by the Root Locus method [24] to support the overshoot and settling time chosen in Table 1. Due to this systematic modeling and tuning procedure, the desired overshoot and settling time can be satisfied (as discussed in Section 5).

\subsection{RTDB Model}

It is known that the transaction arrival rate is useful to model a RTDB in which transaction execution times are usually not known a priori [22]. We have performed a simulation in which the utilization is measured for the increasing transaction arrival rate. To model RTDB dynamics under overload, no feedback control, admission control, or QoS degradation is applied in these experiments. (A detailed description of our simulation settings is given in Section 5.) In Figure 1, the utilization increases and saturates at $100 \%$ as the average arrival rate, indicated by tps (transactions per second), increases. Notably, the success ratio sharply decreases as the arrival rate further increases after the utilization is saturated due to the severe data/resource contention. Thus, it is necessary to avoid the saturation by controlling the arrival rate.

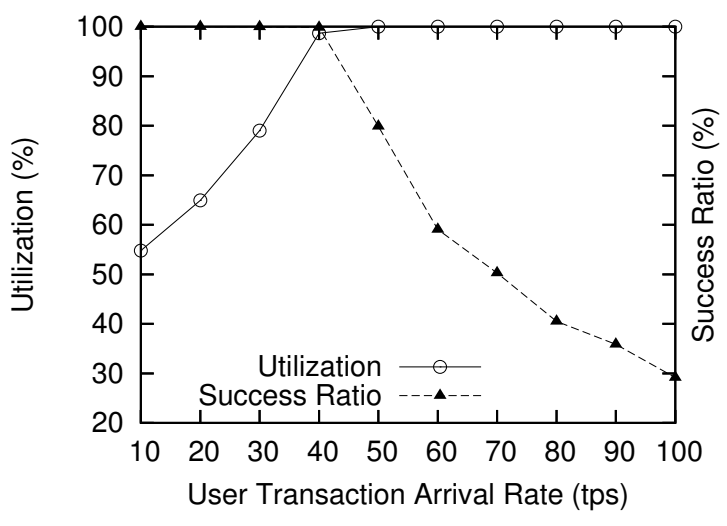

Figure 1. Average Utilization

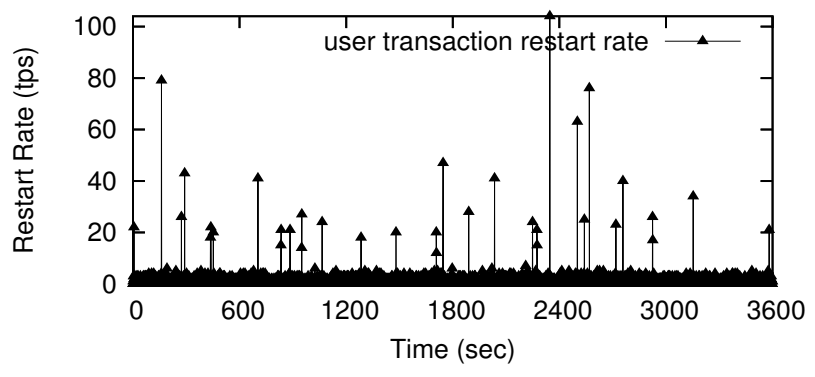

Figure 2. User Transaction Restart Rate

High data contention can also increase the utilization due to many transaction aborts and restarts, causing database thrashing in an extreme case [20]. Figure 2 shows the restart 


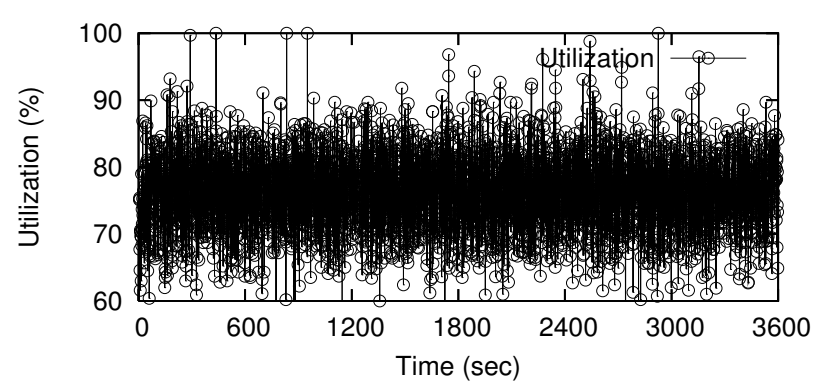

Figure 3. Transient Utilization

rate when the average arrival rate is only 30 tps at which the average utilization is below $80 \%$ as shown in Figure 1. In Figure 2, the transaction restart rate is often greater than 20 tps and it even reaches 110 tps. From these experiments, we observe that it is critical to consider not only the arrival rate $\lambda$ but also the restart rate $\lambda_{r}$. From Figures 1 and 2, we observe that the maximum transient total arrival rate $\lambda^{\prime}=\lambda+\lambda_{r}=30$ tps +110 tps $=140$ tps when $\lambda$ is only 30 tps. For the same arrival rate $30 \mathrm{tps}$, the transient utilization is shown in Figure 3. Although the average utilization is only $79.02 \%$ as shown in Figure 1, the transient utilization widely fluctuates saturating at $100 \%$ several times as shown in the figure. Thus, a system model only based on the arrival rate such as the one used in [22] can involve large errors. If a RTDB model does not consider the restart rate in addition to the arrival rate, too many transactions can run concurrently, further increasing data/resource contention. Consequently, the success ratio could be decreased drastically.

To address the problem, we propose a new utilization control model for RTDBs by considering both the arrival rate and the restart rate. Specifically, we construct a RTDB model based on the ARX (Auto Regressive eXternal) model $[24,9]$ that are widely used to model computational system dynamics $[9,15,3,19,23]$. The ARX model expresses the next output based on the $n$ previous system inputs and outputs where $n(\geq 1)$ determines the system order. Specifically, we model the relationship between the total arrival rate $\lambda^{\prime}(k)$ and utilization $u(k)$ at the $k^{t h}$ sampling period over the sampling history in the discrete time domain as follows:

$$
u(k)=\sum_{i=1}^{n}\left\{a_{i} u(k-i)+b_{i} \lambda^{\prime}(k-i)\right\}
$$

where the total arrival rate $\lambda^{\prime}(k-i)=\lambda(k-i)+\lambda_{r}(k-i)$, i.e., the sum of the transaction arrival rate and restart rate measured at the $(k-i)^{t h}$ sampling period. By considering both the new transaction arrivals and the aborts/restarts of the transactions already in the system, our model can effectively express RTDB dynamics. The model parameters, i.e., $a_{i}$ 's and $b_{i}$ 's in Eq 4, can be derived via system identifica- tion $[9,24]$ discussed next.

\subsection{System Identification}

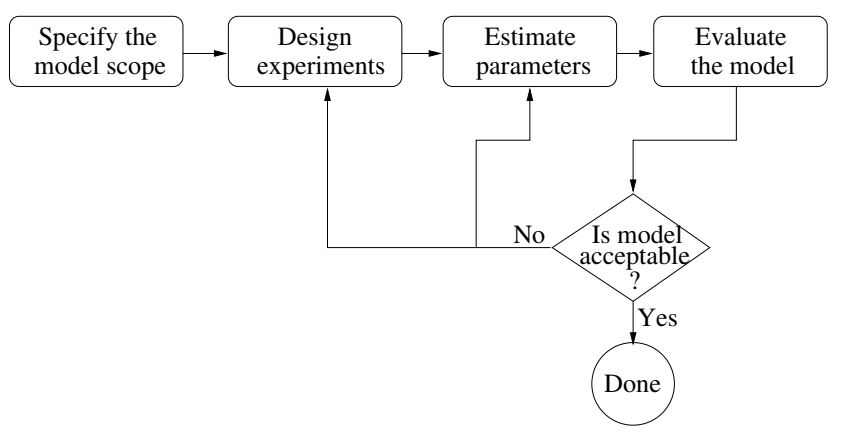

Figure 4. System Identification Steps

In this subsection, the procedure of the system identification based on the RTDB model described in the previous subsection is presented. Figure 4 illustrates a wellestablished five step procedure for black-box modeling $[24,9]$. We follow this procedure to identify the system dynamics by finding and verifying the model parameters in Eq 4 in a systematic manner as follows.

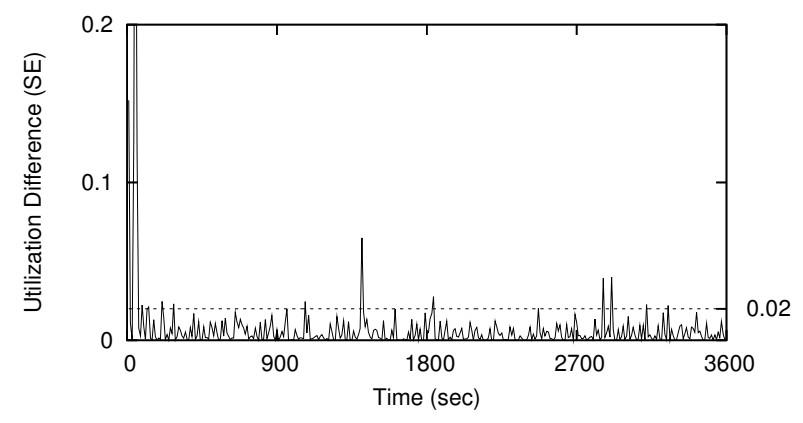

Figure 5. Model Verification

Step 1: Specify the model scope. To see the effect of the arrival/restart rate on the utilization, we have observed how the RTDB utilization changes for the increasing arrival rate as discussed in the previous subsection. From Figure 1, we observe that the utilization increases in a nearly linear fashion between $\lambda=10$ tps and $\lambda=40$ tps. Once $\lambda>40$ tps, the utilization gets saturated as shown in the figure. Hence, we select the arrival rate range [10 tps, 40 tps] as our model scope, since standard linear control theoretic techniques can be applied to identify the system model within this range of the arrival rates. Specifically, we identify the relationship between the total arrival rate and utilization within this scope. (A more detailed discussion is given in the Steps 2 and 3.) In addition, we have to determine the order of the ARX model (Eq 4). A higher order model can generally 
(but not necessarily) improve the accuracy of the model for more complexity [9]. Thus, we have constructed four different models: $1^{\text {st }}, \ldots, 4^{\text {th }}$ order models. (A more detailed discussion is given in the Step 4.)

Step 2: Design experiments. In this step, we collect system dynamics data to estimate model parameters, i.e., $a_{i}$ 's and $b_{i}$ 's in Eq 4. Although the model scope is specified in the Step 1, the variability of the transient utilization is a remaining issue to deal with. In Figure 3, for example, the transient utilization considerably fluctuates for a moderate workload where $\lambda=30$ tps that results in the approximately $79 \%$ average utilization (Figure 1). To smooth the variability of the utilization due to stochastic workloads and dynamic data contention, representative workloads and a large number of system dynamics data $-\lambda^{\prime}$ and $u$ in our approach - should be used and collected [24, 9]. To address this issue, we use well-accepted real-time transaction processing workloads, similar to $[31,5,10]$. For unbiased parameter estimation, we uniformly change the arrival rate $\lambda$ within the range [10tps, 40tps] specified in the Step 1 at every 10 s in which approximately 100 (1000) transactions newly arrive when $\lambda=10$ (100) tps. To collect a large number of performance data, we run a simulation for 60 minutes and obtain $\lambda^{\prime}$ and $u$ at every 10s.

Step 3: Estimate the model parameters. We apply the least square regression method [24, 9] to estimate model parameters in Eq 4 to minimize the sum of the squared prediction errors. Specifically, the prediction error in our model $e(k)=u(k)-u^{\prime}(k)$, where $u(k)$ is the utilization measured at the $k^{t h}$ prediction period and $u^{\prime}(k)$ is the utilization predicted by Eq 4 using the estimated model parameters available at the $k^{t h}$ period. The least square regression method iteratively estimates the model parameters at each sampling instant to minimize the sum of the squared estimation errors $\sum_{k=1}^{m} e(k)^{2}$ where $m$ is the number of the sample instances. We apply the least square regression method to the four different orders of models to compare their accuracy as follows.

Step 4: Evaluate the model. Once the model parameters are estimated from the Step 3, we need to evaluate whether or not system dynamics are well captured by the model. In Figure 5, we plot the squared error (SE) $e(k)^{2}$ at every $10 \mathrm{~s}$ to evaluate the accuracy of the $2^{n d}$ order model. As shown in the figure, the SE is below 0.02 for most of the time. The corresponding root mean square error (RMSE), which is a common correctness metric for system identification [24, 9], is: $R M S E=\sqrt{\sum_{i=1}^{360} e(i)^{2} / 360}=0.0852$, while $R M S E^{\prime}=\sqrt{\sum_{i=181}^{360} e(i)^{2} / 180}=0.0685$ without the initial spikes of errors. The $1^{\text {st }}$ order model has shown the highest error, while the $3^{\text {rd }}$ and $4^{\text {th }}$ order models have not improved the accuracy considerably. Thus, we have selected the second order model due to its high accuracy for less complexity compared to the higher order models. This selection complies with the rule of thumb recommending to select the lowest order model with good accuracy [9].

Step 5: Determine if the model is acceptable. This step determines whether or not the chosen model is acceptable. In this paper, we accept the second order model, since it effectively captures system dynamics as shown in Figure 5. Further, our feedback control scheme based on the second model has achieved good performance. (The detailed performance results will be discussed in Section 5.) The final second order model derived via this five step procedure describes the relationship between $\lambda^{\prime}$ and $u$ in the discrete time domain as follows:

$$
\begin{array}{r}
u(k)=0.5092 u(k-1)+0.2187 u(k-2)+ \\
0.01175 \lambda^{\prime}(k-1)-0.00438 \lambda^{\prime}(k-2)
\end{array}
$$

\subsection{Utilization Controller Design}

As shown in Figure 6, the utilization controller computes the control signal $\Delta \lambda^{\prime}$ based on the error, which is the difference between the target utilization $U_{t}$ and the utilization measured at a sampling instant. Specifically, $\Delta \lambda^{\prime}$ is the control signal by which the RTDB needs to decrease (or increase) $\lambda^{\prime}$ to achieve the desired utilization $U_{t}$ when overloaded (or underutilized).

As the first step to designing the utilization controller, the transfer function of the RTDB is derived by taking the $z$-transform [24, 9] of Eq 5 to describe the relationship between $\lambda^{\prime}$ and $u$ such that the transfer function can be expressed in an algebraic manner:

$$
\begin{aligned}
U(z)= & 0.5092 z^{-1} U(z)+0.2187 z^{-2} U(z)+ \\
& 0.01175 z^{-1} \Lambda^{\prime}(z)-0.00438 z^{-2} \Lambda^{\prime}(z)
\end{aligned}
$$

where $U(z)$ is the $z$-transform of $u(k)$ and $\Lambda^{\prime}(z)$ is the $z$ transform of $\lambda^{\prime}(k)$. After some algebraic manipulation, we get the following transfer function:

$$
G(z)=\frac{U(z)}{\Lambda^{\prime}(z)}=\frac{0.01175 z-0.00438}{z^{2}-0.5092 z-0.2187}
$$

Given the transfer function in $\mathrm{Eq} \mathrm{7,} \mathrm{we} \mathrm{can} \mathrm{design} \mathrm{the}$ utilization controller using a standard controller such as a PID (Proportional-Integral-Derivative) controller [24, 9]. In this paper, we use a PI controller for the following reasons. A P controller cannot remove the steady state error [24, 9]. We have verified this phenomenon via simulation in which the tested $\mathrm{P}$ controllers failed to support the target utilization. An integral controller can be combined with a P controller to eliminate the non-zero steady-state error [24, 9]. A derivative controller could decrease the settling time by adjusting the control input based on changes in control errors. However, a weakness of derivative control is the sensitivity 


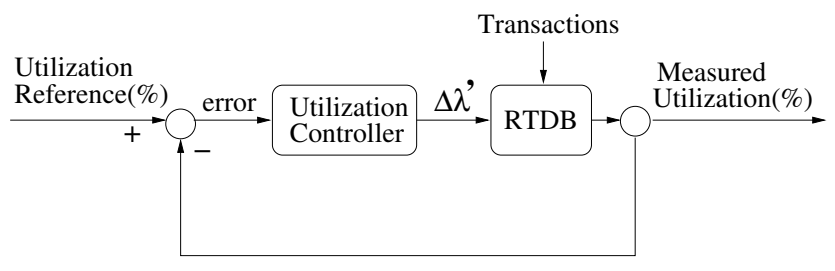

Figure 6. Utilization Control Loop

to noise $[24,9]$. Since RTDB workloads are stochastic involving transaction aborts and restarts, we do not use a D controller in this paper.

The PI control law for our utilization controller is:

$$
\Delta \lambda^{\prime}(k)=K_{P}\left[e(k)+K_{I} \sum_{i=1}^{k} e(k)\right]
$$

where the error $e(k)=U_{t}-u(k)$ at the $k^{t h}$ sampling period. As shown in Figure 6, $e(k)$ is the input to the utilization controller that computes the control signal $\Delta \lambda^{\prime}$, i.e., the required $\lambda^{\prime}$ adjustment at the $k^{t h}$ sampling period, based on $e(k) . K_{P}$ and $K_{I}$ are the proportional and integral control gains. The gains should be chosen carefully to support the desired average and transient performance specified in Table 1. Eq 8 can be rewritten in a more efficient form:

$$
\Delta \lambda^{\prime}(k)=\Delta \lambda^{\prime}(k-1)+K_{P}\left[\left(K_{I}+1\right) e(k)-e(k-1)\right]
$$

The transfer function of a PI controller can be obtained by taking the $z$-transform of Eq 9 to describe the relation between the controller input $e(k)$ and the controller output $\Delta \lambda^{\prime}(k)$ :

$$
C(z)=\frac{\alpha(z-\beta)}{z-1}
$$

where $\alpha=K_{P}\left(K_{I}+1\right)$ and $\beta=1 /\left(K_{I}+1\right)$.

To support the desired control performance summarized in Table 1 by tuning $K_{P}$ and $K_{I}$, we need to find the closedloop transfer function. In control theory, the closed loop transfer function can be derived using the transfer functions of the controller and the controlled system such as the RTDB $[24,9]$. Given the RTDB transfer function $G(z)$ (Eq 7) and PI controller transfer function $C(z)$ (Eq 10), the closed loop transfer function $F_{R}(z)$ is [24, 9]:

$$
F_{R}(z)=\frac{C(z) G(z)}{1+C(z) G(z)}
$$

Given this closed loop transfer function, we use the Root Locus method $[9,24]$ to graphically tune the PI controller in Matlab. In this way, we can locate the closed-loop poles to support the desired performance specified in Table 1. The exact location of the closed-loop poles are -0.289 and $0.876 \pm 0.053 i$. (The corresponding $K_{P}=0.8$ and $K_{I}=4$.) Since the poles are inside the unit circle, the closed-loop system is stable.

\section{Success Ratio Management via Utilization Control}

This section describes the way the key system components such as the transaction scheduler (TS), concurrency controller (CC), admission controller, and QoS manager interact based on the control signal computed at every sampling period.

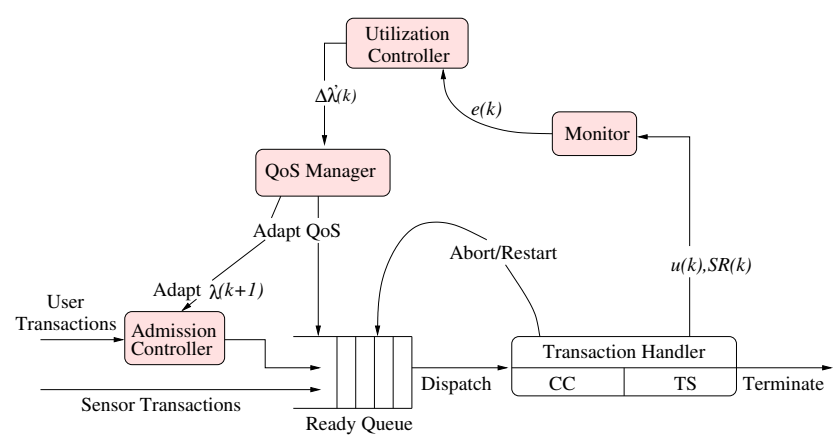

Figure 7. Real-Time Database Architecture

Figure 7 illustrates the RTDB architecture designed to support a high success ratio. There are two types of transactions. Sensor transactions periodically update the sensor data in the RTDB to reflect the current real-world status. User transactions arrive aperiodically. They can read sensor data and read/write non-sensor data as discussed before. The Transaction Handler schedules transactions in an EDF (Earliest Deadline First) [14] fashion. It supports both pessimistic and optimistic concurrency protocols: two phase locking high priority (2PL-HP) [1] and Wait-50 [8]. In 2PL$\mathrm{HP}$, which is pessimistic, all data conflicts are immediately resolved by aborting lower priority transactions. Hence, 2PL-HP is free of priority inversion. Wait-50 is a well known optimistic concurrency control protocol, in which a validating transaction can commit when it has a higher priority than more than half of the transactions conflicting with itself. We consider both 2PL-HP and Wait-50, since the performance of real-time concurrency control mechanisms varies depending on several factors such as the degree of data/resource contention or deadline semantics [12]. The Monitor measures the system status such as the utilization $u(k)$ and success ratio $S R(k)$ at the $k^{t h}$ sampling period. It can dynamically adjust $U_{t}$ based on the success ratio as discussed in Section 2. Based on the difference between the target utilization and monitored utilization, the Utilization Controller computes $\Delta \lambda^{\prime}(k)$ that is the $\lambda^{\prime}$ adjustment required to support $U_{t}$.

As described in Figure 8, the QoS Manager and Admission Controller adapt the workload according to the control signal. Note that QoS degradation is applied before admission control to improve the success ratio. When the system 
1. At the $k^{t h}$ sampling period, the Monitor receives $u(k)$ and computes the utilization error $e(k)=U_{t}-u(k)$.

2. Based on $e(k)$, the Utilization Controller computes the control signal $\Delta \lambda^{\prime}(k)$. Under overload, $\Delta \lambda^{\prime}(k)<$ 0 requiring the RTDB to reduce data/resource contention. If $\Delta \lambda^{\prime}(k) \geq 0$, skip the Step 3 .

3. When $\Delta \lambda^{\prime}(k)<0$, the QoS Manager degrades the QoS of the transactions in the system to support the desired utilization by applying the milestone approach [13], similar to [29]. After degrading transaction $T_{i}$, adjust $\Delta \lambda^{\prime}(k)=\Delta \lambda^{\prime}(k)+1-Q\left(T_{i}\right)$ where $Q\left(T_{i}\right)$ is the QoS level of $T_{i}$ computed using Eq 2. Perform all possible QoS degradation until $\Delta \lambda^{\prime}(k) \geq 0$ or no transaction can be degraded anymore.

4. Apply admission control to the transactions arriving during the $(k+1)^{t h}$ sampling period. In this period, admit no more than $\lambda_{a}=\lambda(k)+\Delta \lambda^{\prime}(k)$ incoming transactions.

\section{Figure 8. FACT Procedure}

is overloaded due to data/resource contention, $\Delta \lambda^{\prime}(k)<0$. In this case, a subset of transactions in the system can be degraded. When transaction $T_{i}$ is degraded, its QoS level $Q\left(T_{i}\right)$ (defined in Eq 2) monotonically decreases as more optional parts are skipped, similar to [13, 29]. Since $1-Q\left(T_{i}\right)$ represents the fraction of the original transaction that is not executed due to the QoS degradation, the QoS degradation is conceptually equivalent to decreasing the arrival rate by $1-Q\left(T_{i}\right)$. Data/resource contention can be decreased in this way, because a degraded transaction will access a reduced number of data doing less arithmetic/logical computation. Hence, we increase $\Delta \lambda^{\prime}(k)$ by $1-Q\left(T_{i}\right)$ after degrading $T_{i}$ to $Q\left(T_{i}\right)$. We repeat the QoS adaptation until $\Delta \lambda^{\prime}(k) \geq 0$ or no transaction can be degraded anymore.

In the next sampling period, i.e., $(k+1)^{t h}$ sampling period, FACT admits no more than $\lambda_{a}$ transactions where $\lambda_{a}=\lambda(k)+\Delta \lambda^{\prime}(k)$. Thus, FACT admits more transactions in the next period when $\Delta \lambda^{\prime}(k)>0$. In contrast, FACT admits a smaller number of transactions in the next period if $\Delta \lambda^{\prime}(k)<0$ to avoid overloads by reducing the arrival rate. Especially in this case, FACT admits incoming transaction $T_{i}$ with the degraded QoS $Q\left(T_{i}\right)$ and consider that only $Q\left(T_{i}\right)(<1)$ transaction is admitted. In this way, it can admit more transactions to enhance the success ratio under overload.

\section{Performance Evaluation}

For performance evaluation, we have built a RTDB simulator to model the RTDB architecture depicted in Figure 7. We have performed all the experiments described in this section for both 2PL-HP [1] and Wait-50 [8]. For Wait50, we have performed separate RTDB modeling, system identification, and controller tuning described in this paper. However, we only present the results under 2PL-HP due to space limitations. Generally, FACT has shown a slightly better performance with Wait-50. We run one simulation for 20 simulated minutes. Each performance data presented here is the average of 10 simulation runs. Since the $90 \%$ confidence intervals are below $3 \%$, we do not plot them for the clarity of presentation. Further, we ignore the overhead of feedback control, because it is known to consume less than $1 \%$ CPU utilization [19].

A number of RTDB applications, e.g., agile manufacturing, traffic control, and target tracking, monitor the real world status via periodic sensor readings, while processing user transactions in a timely fashion using sensor and non-sensor data. Our workloads are similar to the workloads commonly found in the RTDB literature including $[31,5,10]$. The workloads of [31] are derived from air traffic control. The workloads of [10] are modeled after stock trading, while the workloads of [5] are similar to [31, 10] except data imprecision considered in their work. A more detailed discussion follows.

Data and Sensor Transactions. There are 1000 sensor data and 1000 non-sensor data in the (simulated) RTDB. The sensor data objects are periodically updated by 1000 independent sensor transactions. Sensor transaction $S T_{i}$ 's period $P_{i}$ is uniformly distributed in a range (100ms, 50s). The relative deadline of $S T_{i}$ is equal to its period $P_{i} . S T_{i}$ 's execution time is uniformly distributed in a range $(3 \mathrm{~ms}$, $6 \mathrm{~ms})$. The total utilization required by the sensor transactions is approximately $50 \%$. All periodic sensor transactions meet their deadlines to support the basic data temporal consistency requirement specified by the absolute validity intervals [25].

User Transactions. The execution time $E T_{j}$ of user transaction $T_{j}$ is uniformly distributed in a range $(5 \mathrm{~ms}, 20 \mathrm{~ms})$ and $T_{j}$ 's deadline $D_{j}=T_{j}$ 's arrival time + slack factor (SF) $\times E T_{j}$ where the $\mathrm{SF}$ is uniformly distributed in a range (10, 20). The number of $T_{j}$ 's data accesses is proportional to $E T_{j}$. If $E T_{j}$ is $5 \mathrm{~ms}$, for example, $T_{j}$ performs $5 \mathrm{read} / \mathrm{write}$ operations (and computations). An operation in $T_{j}$ accesses a sensor data with probability 0.4 . Thus, user transaction $T_{j}$ reads 2 sensor data items and writes 3 non-sensor data items when $E T_{j}=5 \mathrm{~ms}$. For $T_{j}$, we set the conditional probability $\mathrm{P}($ write $\mid$ non-sensor data access $)=1$ to create data conflicts, which can stress FACT and the tested baselines. Because a sensor data item $i$ is updated by a dedicated sen- 
sor transaction $S T_{i}$ and user transactions can only read sensor data, we do not need full concurrency control for sensor data. Instead, we use short latches to prevent user transactions from reading sensor data, while they are updated, similar to [31]. Hence, neither sensor nor user transactions are aborted/restarted due to sensor data accesses. To model high data contention, we follow the $90-10$ access pattern, in which $90 \%$ of RTDB accesses are directed to the $10 \%$ of the data in the RTDB. Note that FACT and the tested baselines are unaware of $E T_{j}$ and the data access pattern. A transaction consists of a mandatory part and optional parts, similar to [29]. When $T_{j}$ is fully degraded, we assume that $E T_{j}$ and $Q\left(T_{j}\right)$ are reduced by $30 \%$ to $60 \%$ in a uniform random manner to consider the fact that different transactions may allow different degrees of QoS degradation. User transactions follow the Poisson arrival pattern. The average arrival rate $\lambda$ is varied from 10 tps to 100 tps. The load applied to the RTDB is approximately $60 \%$ when $\lambda=10$ tps and $170 \%$ when $\lambda=100$ tps.

Baselines. Given the sensor and user transaction workload settings, we use the following baseline approaches that have been widely used for performance comparisons in RTDBs [5, 10, 11]. (i) Admit-All admits all incoming transactions regardless of the current system status. It does not degrade the QoS either. (ii) Ad-Hoc rejects all incoming user transactions when the utilization is higher than the target utilization $U_{t}$, similar to $[11,20]$. Unlike FACT, $U_{t}$ is fixed at 95\% for Ad-Hoc to admit as many transactions as possible to enhance the success ratio. Further, it does not degrade the QoS. We consider this baseline, since admission control is known to be very effective to deal with overloads in database systems $[5,11,10,30]$. In addition, we consider (iii) FACT without $Q o S$, which applies the control theoretic approach and admission control method described in this paper without adapting QoS. Note that existing work on feedback control of RTDB performance including $[4,5,10]$ assume knowledge of estimated transaction execution times, whereas FACT does not. Thus, they are not directly comparable to FACT. FACT could also perform better when estimated execution times are known in certain cases. A thorough investigation is reserved for future work.

Performance Results. Figure 9 illustrates the average utilization for the increasing average arrival rate $\lambda$. The average utilization of Admit-All gradually increases and saturates when $\lambda \geq 60$ tps, overloading the RTDB. Ad-Hoc supports the desired $95 \%$ average utilization for $\lambda \geq 60$ tps, because it drops every incoming transaction whenever the utilization $>95 \%$. However, its success ratio quickly drops as $\lambda$ increases due to aggressive admission control. (A detailed discussion of the success ratio will follow shortly.) Further, its transient utilization significantly fluctuates, since it does not continuously control the utilization in a feedback loop as FACT does. FACT without QoS closely achieves the de-

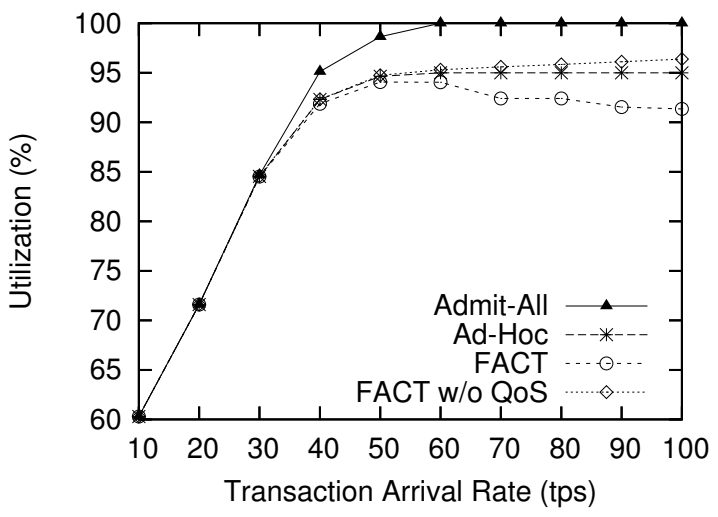

Figure 9. Average Utilization

sired $95 \%$ utilization when $\lambda \geq 60$ tps. It also supports the desired transient performance specified in Table 1. (The transient performance results of Ad-Hoc, Admit-All, and FACT without QoS are not included due to space limitations.) By applying QoS management and admission control according to the control signal, FACT successfully supports the target utilization $U_{t} \in[90 \%, 95 \%]$ for $\lambda \geq 40$ tps. FACT shows flexible utilization management in the specified range of $U_{t}$ to improve the success ratio by increasing $U_{t}$ within the bound when the success ratio $\geq 99 \%$, while avoiding overshoots and resulting saturations by decreasing $U_{t}$ under overload as discussed in Section 2. From the figure, observe that FACT reduces its utilization set-point $U_{t}$ as $\lambda$ exceeds 60 tps. As a result, the average utilization of FACT becomes $91.35 \%$ when $\lambda=100$ tps.

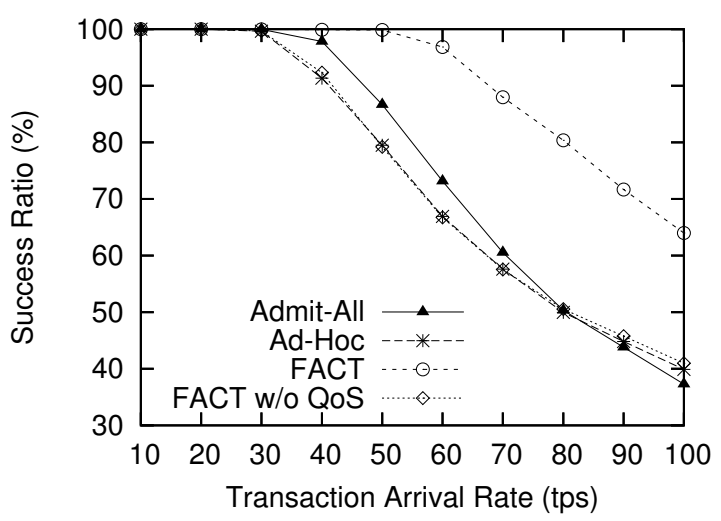

Figure 10. Average Success Ratio

In Figure 10, FACT's success ratio is $100 \%$ when $\lambda \leq 50$ tps. Its success ratio is considerably higher than Admit-All and Ad-Hoc when $\lambda \geq 60$ tps. It enhances the success ratio by $20 \%$ - $30 \%$ compared to Admit-All and Ad-Hoc by dynamically adapting the system behavior via feedback control, QoS degradation, and admission control consider- 
ing the current data/resource contention. Ad-Hoc generally shows the lowest success ratio among the tested approaches due to its aggressive admission control. For $\lambda \geq 90$ tps, due to admission control, its success ratio is slightly higher than Admit-All, which is severely overloaded; however, the difference is less than 3\%. The success ratio of FACT without QoS is slightly higher than Ad-Hoc, but its transient utilization is stable unlike Admit-All and Ad-Hoc showing a lot of fluctuations. Thus, FACT without QoS could provide more reliable real-time data service than Admit-All and Ad-Hoc.

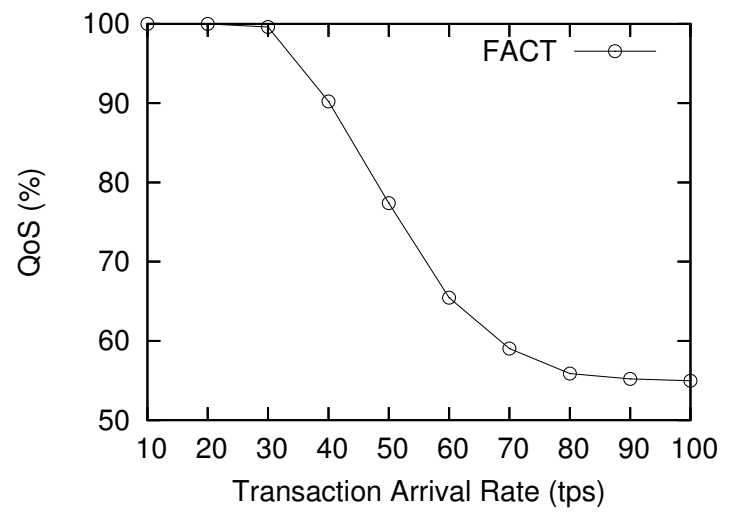

Figure 11. Average QoS

The average QoS supported by FACT is shown in Figure 11. FACT gracefully degrades the QoS as $\lambda$ increases. Its QoS is initially $100 \%$ and reduces to $54.98 \%$ when $\lambda=100$ tps. Since Admit-All and Ad-Hoc do not support the QoS adaptation, the average QoS is $100 \%$ for every arrival rate, causing the poor success ratio especially at high arrival rates as shown in Figure 10.

Figure 12 shows the transient performance of FACT for the relatively high arrival rates that result in more than the 90\% average utilization (Figure 9). When $\lambda<40$ tps, FACT has shown no overshoot of the transient utilization. (We do not plot the transient performance for $\lambda<40$ tps due to space limitations.) Since Admit-All fails to maintain $U_{t}$ and the success ratio of Ad-Hoc is generally lower than Admit-All, we only show FACT's transient utilization and QoS. We consider the RTDB is in the steady state when the transient utilization is between [90\%, 95\%] specified in Table 1. In Figure 12, the utilization is never saturated at $100 \%$ after the system becomes stable for all the arrival rates. The settling time $K_{s}$ is less than the required $250 \mathrm{~s}$ (Table 1) for all the tested arrival rates. Specifically, $K_{s}=$ $14 \mathrm{~s}, 30 \mathrm{~s}, 213 \mathrm{~s}$ and 249s for $\lambda=40 \mathrm{tps}, 60 \mathrm{tps}, 80 \mathrm{tps}$, and 100 tps, respectively. After being stabilized, the transient utilization mainly stays between [89\%, 96\%], closely satisfying the transient performance requirements. From the figure, we also observe that FACT dynamically degrades the QoS to avoid overload. Especially, this phenomenon be-



(a) $\lambda=40$ tps

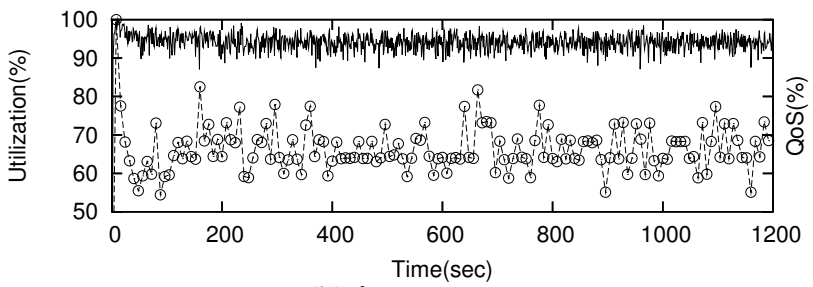

(b) $\lambda=60$ tps

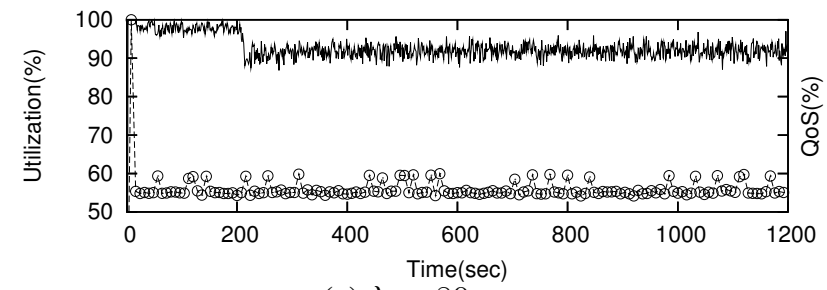

(c) $\lambda=80$ tps

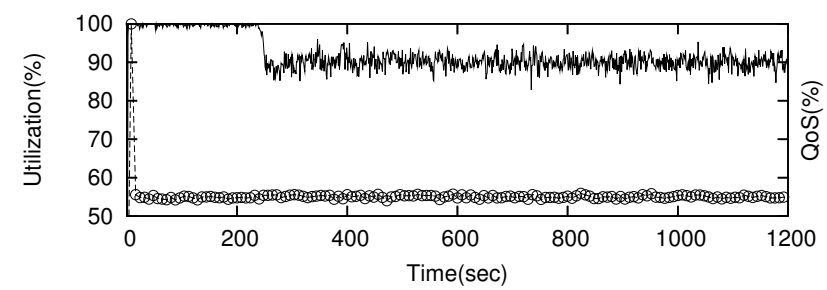

(d) $\lambda=100$ tps

\section{Figure 12. Transient Utilization and QoS of FACT}

comes more evident as $\lambda$ increases. Figures 12(a) and 12(b) show some QoS fluctuations. This is because FACT dynamically adapts $U_{t}$ to increase the success ratio whenever there is a chance. As a result, the fraction of transactions (incoming or already in the system) to be degraded may vary. In addition, we measure the QoS for timely transactions that commit within their deadlines. Thus, transactions degraded at a certain time can commit at different times, introducing variations in the measured QoS. (More advanced QoS management is reserved for future work to reduce QoS variations.) In contrast, the QoS is relatively stable for $\lambda=80$ tps and $\lambda=100$ tps as shown in Figure 12(c) and Figure 12(d). This is because the RTDB does not increase $U_{t}$ and degrades most transactions to avoid overload. From 
these results, we can conclude that FACT can support both the required average and transient utilization, while substantially improving the success ratio, via feedback control, QoS management, admission control, with no knowledge of the transaction execution time.

\section{Related Work}

Feedback control has been applied to real-time task scheduling to manage the utilization and deadline miss ratio given unpredictable loads $[16,18]$. Most existing RTDB work on feedback control including $[4,5,10]$ has adopted control theoretic models similar to $[16,18]$ to manage the transaction timeliness and data freshness/imprecision. However, these work do not consider database-specific transaction aborts/restarts for RTDB modeling. They also require a priori knowledge of (estimated) transaction execution times, which are difficult to predict in RTDBs $[25,26]$.

Möenkeberg et al [20] observe that databases thrash under high data contention, which may cause many deadline misses in RTDBs. To avoid thrashing due to data contention, they measure data conflicts and dynamically adjust admission control to prevent thrashing, while improving the response time and throughput. However, transaction deadlines, data temporal consistency, and formal control theoretic approaches are not considered in their work.

Schroeder et al [27] propose a feedback-based approach to determine an appropriate multi-programming level, i.e., the number of transactions allowed to concurrently execute, in a database system to avoid either underutilization or overload. However, in their work, no mathematical database model based on control theory is constructed. The controller design process does not follow standard control theoretic approaches and stability analysis is not performed. They do not consider transaction deadlines and data temporal consistency critical in RTDBs. Also, QoS adaptation is not considered.

Our QoS adaptation scheme is similar to APPROXIMATE [29] that applies the milestone approach [13] to monotonically improve the accuracy of query answers, while returning an approximate answer to meet the query deadline. Another option is database sampling [21] in which more data can be sampled to improve the accuracy of answers to real-time queries. These work, however, do not consider feedback control.

System identification has been used to model dynamics of software systems including the Apache web server, MPEG player, real-time middleware, and email server $[15,3,19,23]$. These work, however, are not directly applicable to RTDBs, because these systems do not consider RTDB-specific issues such as transaction aborts/restarts and the quality of query/transaction processing [29]. Thus, the control model, QoS metrics, and the QoS management techniques are different.

\section{Conclusions}

RTDBs can be employed in a number of data-intensive real-time applications such as agile manufacturing and target tracking. It is challenging to manage the RTDB performance due to data/resource conflicts. In this paper, we propose a new RTDB control model that effectively captures RTDB-specific data/resource contention requiring no knowledge of transaction execution times. Based on the model, we design and tune a utilization controller. According to the control signal, i.e., the required total arrival rate adjustment, our QoS management and admission control schemes adapt the workload, if necessary, to support the target utilization. In a simulation study, we show that our approach can support the target utilization, while substantially improving the success ratio compared to the tested baselines under overload. In the future, we will investigate a more advanced RTDB control model and QoS adaptation/admission control techniques to further improve the quality of realtime data service.

\section{References}

[1] R. Abbott and H. Garcia-Molina. Scheduling Real-Time Transactions: A Performance Evaluation. ACM Transactions on Database System, 17:513-560, 1992.

[2] T. F. Abdelzaher and N. Bhatti. Adaptive Content Delivery for Web Server QoS. In International Workshop on Quality of Service, June 1999.

[3] L. Abeni, L. Palopoli, G. Lipari, and J. Walpole. Analysis of a Reservation-Based Feedback Scheduler. In Proceedings of the 23rd IEEE Real-Time Systems Symposium, page 71, 2002.

[4] M. Amirijoo, N. Chaufette, J. Hansson, S. H. Son, and S. Gunnarsson. Generalized Performance Management of Multi-Class Real-Time Imprecise Data Services. In Proceedings of the 26th IEEE International Real-Time Systems Symposium, pages 38-49, 2005.

[5] M. Amirijoo, J. Hansson, and S. H. Son. Specification and Management of QoS in Real-Time Databases Supporting Imprecise Computations. IEEE Transactions on Computers, 55(3):304-319, 2006.

[6] J. Baulier, P. Bohannon, S. Gogate, C. Gupta, S. Haldar, S. Joshi, A. Khivesera, H. Korth, P. Mcilroy, J. Miller, P. P. S. Narayan, M. Nemeth, R. Rastogi, S. Seshadri, A. Silberschatz, S. Sudarshan, M. Wilder, and C. Wei. DataBlitz Storage Manager: Main Memory Database Performance for Critical Applications. In ACM SIGMOD - Industrial Session: Database Storage Management, 2000.

[7] R. Elmasri and S. B. Navathe. Fundamentals of Database Systems. Addison Wesley, 4th edition, 2003. 
[8] J. R. Haritsa, M. J. Carey, and M. Livny. Data access scheduling in firm real-time database systems. Real-Time Systems, 4(3):203-241, 1992.

[9] J. L. Hellerstein, Y. Diao, S. Parekh, and D. M. Tilbury. Feedback Control of Computing Systems. A John Wiley and Sons, Inc., Publication, 2004.

[10] K.-D. Kang, S. H. Son, and J. A. Stankovic. Managing Deadline Miss Ratio and Sensor Data Freshness in RealTime Databases. IEEE Transactions on Knowledge and Data Engineering, 16(10):1200-1216, 2004.

[11] S. Kim, S. H. Son, and J. A. Stankovic. Performance Evaluation on a Real-Time Database. In IEEE Real-Time Technology and Applications Symposium, 2002.

[12] J. Lee and S. H. Son. Performance of Concurrency Control Algorithms for Real-Time Database Systems. In V. Kumar, editor, Performance of Concurrency Control Mechanisms in Centralized Database Systems. Prentice Hall, 1996.

[13] K. J. Lin, S. Natarajan, and J. W. S. Liu. Imprecise Results: Utilizing Partial Computations in Real-Time Systems. In Real-Time System Symposium, December 1987.

[14] C. L. Liu and J. W. Layland. Scheduling Algorithms for Multiprogramming in a Hard-Real-Time Environment. Journal of the ACM, 20(1):46-61, 1973.

[15] C. Lu, T. F. Abdelzaher, J. A. Stankovic, and S. H. Son. A Feedback Control Approach for Guaranteeing Relative Delays in Web Servers. In Proceedings of the Seventh RealTime Technology and Applications Symposium, page 51, 2001.

[16] C. Lu, J. Stankovic, T. Abdelzaher, G. Tao, S. H. Son, and M. Marley. Performance Specifications and Metrics for Adaptive Real-Time Systems. In Real-Time Systems Symposium, Orlando, Florida, November 2000.

[17] C. Lu, J. Stankovic, G. Tao, and S. Son. Design and Evaluation of a Feedback Control EDF Algorithm. In Real-Time Systems Symposium, December 1999.

[18] C. Lu, J. A. Stankovic, G. Tao, and S. H. Son. Feedback Control Real-Time Scheduling: Framework, Modeling and Algorithms. Real-Time Systems, Special Issue on ControlTheoretical Approaches to Real-Time Computing, 23(1/2), May 2002.

[19] C. Lu, X. Wang, and C. Gill. Feedback Control Real-Time Scheduling in ORB Middleware. In Proceedings of the The 9th IEEE Real-Time and Embedded Technology and Applications Symposium, page 37, 2003.

[20] A. Möenkeberg and G. Weikum. Conflict-Driven Load Control for the Avoidance of Data-Contention Thrashing. In Proceedings of the Seventh International Conference on Data Engineering, pages 632-639, 1991.

[21] G. Ozsoyoglu, S. Guruswamy, K. Du, and W.-C. Hou. TimeConstrained Query Processing in CASE-DB. IEEE Transactions on Knowledge and Data Engineering, pages 865-884, Dec 1995.

[22] H. Pang, M. J. Carey, and M. Livny. Multiclass Query Scheduling in Real-Time Database Systems. IEEE Transactions on Knowledge and Data Engineering, 7(4):533-551, August 1995.

[23] S. Parekh, N. Gandhi, J. Hellerstein, D. Tilbury, T. Jayram, and J. Bigus. Using Control Theory to Achieve Service Level Objectives In Performance Management. Real-Time Systems, 23(1-2):127-141, 2002.
[24] C. L. Phillips and H. T. Nagle. Digital Control System Analysis and Design (3rd edition). Prentice Hall, 1995.

[25] K. Ramamritham. Real-Time Databases. International Journal of Distributed and Parallel Databases, 1(2), 1993.

[26] K. Ramamritham, S. H. Son, and L. C. Dipippo. Real-Time Databases and Data Services. In Real-Time Systems, volume 28, Nov.-Dec. 2004.

[27] B. Schroeder, M. Harchol-Balter, A. Iyengar, E. Nahum, and A. Wierman. How to Determine a Good Multi-Programming Level for External Scheduling. In Proceedings of the 22nd International Conference on Data Engineering, page 60, 2006.

[28] The TimesTen Team. In-Memory Data Management for Consumer Transactions The TimesTen Approach. In ACM SIGMOD, 1999.

[29] S. Vrbsky. APPROXIMATE: A Query Processor that Produces Monotonically Improving Approximate Answers. $\mathrm{PhD}$ thesis, University of Illinois at Urbana-Champaign, 1993.

[30] G. Weikum, C. Hasse, A. Möenkeberg, and P. Zabback. The COMFORT Automatic Tuning Project. Information Systems, 19(5):381-432, 1994.

[31] M. Xiong, K. Ramamritham, J. A. Stankovic, D. Towsley, and R. Sivasankaran. Scheduling Transactions with Temporal Constraints: Exploiting Data Semantics. IEEE Transactions on Knowledge and Data Engineering, 14(5):11551166, September/October 2002. 